\title{
Neutrons, sciences and perspectives
}

The "Société Française de la Neutronique, SFN" (http://www.sfn.asso.fr/) is devoted to promote exchange between the scientific staff from the neutron facilities in France (Laboratoire Léon Brillouin at Saclay and Institut Laue Langevin at Grenoble) and the neutron users from academia and research organisations. SFN is also the French representative in the European Neutron Scattering Association (ENSA) that provides a link to the European and international neutron community.

SFN organizes since 1992 the annual meeting "Journées de la Diffusion Neutronique (JDN)" including, since 1995, a topical School followed by a national conference where the recent results obtained with neutron scattering are exposed and discussed. A series of publications has been the output of the schools and conferences (http://www.neutron-sciences.org). At the JDN19 meeting (in Batz-surMer, June 6-10, 2011) the school was replaced by a longer conference intending to overview the wide variety of scientific disciplines where neutron scattering is a relevant probe.

The meeting in Batz included introductory talks by outstanding invited lecturers aiming to inform about the "state of the art" in scientific domains currently marked as "of prime importance" for academic and social progress. These invited talks covered a wide panel of disciplines: material sciences focusing on specific properties of new functional materials and nanostructures, prospects on sustainable energy, health and food sciences, environmental concerns, etc.... Then a few recognized scientists involved in neutron studies reviewed recent results obtained with neutron techniques in these scientific domains. Finally, each session ended with shorter presentations on specific points, with original results taking advantage of the specific interaction of the neutron probe with condensed matter and of its complementarities with other experimental techniques.

The historical development of neutron scattering started from structural investigations and was further extended to the study of dynamical aspects (https://www.nobelprize.org/nobel_prizes/physics/laureates/1994/). Neutron scattering has made outstanding contributions to reveal on the microscopic level properties of technologically important materials such as plastics, polymers, fibres, liquid crystals, catalysts, proteins, ceramics, metallic alloys, magnets and superconductors as well as to our understanding of fundamental phenomena such as phase transitions, quantum fluids and spontaneous ordering.

In association with the progress of instrumentation and data analysis methodology, it is possible to distinguish the scientific areas for which neutron scattering has become a well established key probe and others where neutrons are complementary and are even possibly still looking for full confirmation of their potential. We can examine the 
contributions of the present issue in this context. In this volume we grouped articles on a wide variety of themes and scientific challenges.

Among the specific properties of neutrons, the high penetration in most materials, the ability to discriminate between isotopes of the same atom, the important interaction with magnetisation and the incoherent scattering make neutron scattering a unique tool for the study of condensed matter, its structure and dynamics, both individual and collective.

Several contributions to this issue demonstrate this wide coverage. For example, the sensitivity of neutrons to microscopic magnetization related to the electronic state of a solid is an indispensable tool for the investigation of static and dynamic properties of magnetic materials. Several contributions illustrate the versatility of neutron probe applied with success to modern problems of magnetism that include magnetic chirality (V. Simonet et al.), frustration (I. Mirebeau and S. Petit), molecular nanomagnets (M. Baker and H. Mutka) and magnetoelectrics (M. Hatnean et al.). The reviews and original results presented here deal with fundamental issues but, in general, longer term prospects of applications provide motivation in addition to pure scientific curiosity. Moreover, the exposed work highlights recent methodological progress with polarization analysis, investigation of excitations in single crystal samples using time-of flight techniques as well as data analysis and modelling.

Another specific strength of neutrons is the investigation of the dynamics of condensed matter on a large scale of characteristic distances, going from atomic to mesoscopic scales . Thanks to neutron scattering, the understanding of vibrational states, structural relaxation of crystalline solids and atomic diffusion can be enlightened. The focus is now on the application of well established methods to more complex systems such as nanoscale carbon materials (S. Rols et al.), supramolecular architectures (A. Desmedt et al.), systems under confinement (J.-M. Zanotti et al.) or diffusion of molecules on surfaces (I. Calvo-Almazan and P. Fouquet).

In the context of structure and dynamics and, the special sensitivity of neutrons with respect to hydrogen and lithium is highlighted in papers concerning proton conduction (P. Colomban and A. Slodczyk), operation of fuel cells (S. Lyonnard and G. Gebel), electrode materials for lithium batteries (C. Masquelier). These presentations also addressed several questions relevant to technological applications, for example in the field of renewable energy sources.

We can also note the versatility of neutrons for systems with spatial length scales beyond the atomic level. The focus is on studies on soft matter and materials of biological interest. The large difference between the coherent scattering lengths of hydrogen and deuterium offers the opportunity of detailed studies where parts of a hydrogenated sample are deuterated, as in polyelectrolytes (J. Combet et al.). Even if Small Angle Neutron Scattering is a central technique (G. Chaboussant et al.), studies on soft matter and biological systems use the full spectrum of neutron techniques (see tutorials review of M. Gradzielski et al. on self-assembled systems and of G. Fragneto on model membranes), including also reflectometry (Y. Corvis et al., A. GuyomardLack et al., N. Brouette et al.), diffraction (F. Sebastiani et al.), time-of-flight and spin echo techniques (D. Bhowmick et al.).

Emerging domains, such as environment sciences (B. Demirdjian et al.), energy technologies (C. Bauer et al.), climatology, geophysics, are using more and in a better way the possibilities of neutron scattering and one purpose of this issue is to call the attention of scientists working in such domains to the possibilities of neutron scattering.

However, the present collection of articles is by no means a comprehensive presentation on the full extent of the capabilities of neutron science. Instead it is like a snapshot demonstrating the progress of scientific output within some of the current topics of importance among the various scientific disciplines profiting of neutron 
techniques. Each paper ends with a brief note 'about the authors and acknowledgements' which emphasizes the extended range of the collaborative effort and the mobility of researchers that characterize neutron science.

The editors are indebted to the numerous colleagues who helped to review the submitted manuscripts and therefore contributed to ensure a high level of scientific standard.

The guest editors:

Etienne Janod, Institut des Matériaux Jean Rouxel de Nantes, organizer of the JDN19 meeting at Batz-sur-Mer

Françoise Leclercq-Hugeux, CNRS-LASIR-Lille, former President of the Société Française de la Neutronique

Hannu Mutka, Institut Laue Langevin, Grenoble José Teixeira, Laboratoire Léon Brillouin, Saclay 